\title{
Laser-modified one- and two-photon absorption: Expanding the scope of optical nonlinearity
}

\author{
David S. Bradshaw and David L. Andrews* \\ School of Chemistry, University of East Anglia, Norwich Research Park, Norwich NR4 7TJ, United Kingdom
}

(Received 26 June 2013; published 4 September 2013)

\begin{abstract}
It is shown that conventional one-photon and two-photon absorption processes can be made subject to nonlinear optical control, in each case significantly modifying the efficiency of absorption, through the effect of a secondary, off-resonant stimulus laser beam. The mechanistic origin of these laser-modified absorption processes, in which the stimulus beam emerges unchanged, is traced to higher-order terms in standard perturbation treatments. These normally insignificant terms become unusually prominent when the secondary optical stimulus is moderately intense. Employing a quantum formulation, the effects of the stimulus beam on one-photon and two-photon absorption are analyzed, and calculations are performed to determine the degree of absorption enhancement, and the form of spectral manifestation, under various laser intensities. The implications of differences in selection rules are also considered and exemplified, leading to the identification of dark states that can be populated as a result of laser-modified absorption. Attention is also drawn to the possibility of quantum nondemolition measurements, based on such a form of optical nonlinearity.
\end{abstract}

DOI: 10.1103/PhysRevA.88.033807

PACS number(s): 42.50.Nn, 42.50.Ct, 42.65.-k, 33.80.-b

\section{INTRODUCTION}

It is usually considered self-evident that conventional, single-photon absorption of light by molecules (or atoms) occurs through individual photons of an appropriate wavelength resonantly interacting with the individual molecules. By extension, multiphoton absorption is equally considered to result from the combined action of two or more photons acting together, in individual molecules with energy levels suitably placed to capture more than one photon at a time. Across the field of absorption spectroscopy, a corollary to this premise is that photons of a wavelength outside the range that the sample molecules directly absorb, by any single-photon or multiphoton process, play no part in the absorption of other, suitably resonant photons.

One of the first challenges to this premise arose on recognition that in a cooperative process, two neighboring absorbers might each absorb an off-resonant photon having equal but opposite shifts from the resonant frequencyan electrodynamic interaction between the two atoms or molecules remediating energy conservation at each location by virtual photon coupling. This prospect was first raised in an early study of absorption from intense broadband radiation, an analysis that was largely overlooked at the time [1]. Although this is a principle that does have wider application, suggesting for example a mechanism for subtle differences in the linewidth of spectra secured by dispersive and Fouriertransform instrumentation, all such effects are undoubtedly weak and they specifically hinge on pairwise interactions between the absorbers.

The following analysis tackles a very different and potentially much more prominent mechanism, one that also provides for off-resonant light to modify the characteristics of absorption, both in the case of single-photon absorption and of two-photon absorption. This has no connection with weak absorption at the far wing extremities of nonresonant radiation, as one might suppose occurs for ultrashort pulses:

\footnotetext{
*d.1.andrews@uea.ac.uk
}

the mechanism upon which we shall focus is valid in regions of explicitly zero overlap between the spectral profiles of the electromagnetic radiation and the excited state. Specifically, it will be shown that absorption rates may be significantly modified by an off-resonant laser beam of sufficient intensity, through nonlinear coupling mechanisms occurring in each atom or molecule individually. The origin of these mechanisms can be traced to higher-order terms in the standard perturbation treatment of absorption which, although normally insignificant, become unusually prominent under the conditions we describe. The full nature of this process is described in detail, and its characteristics are analyzed using a quantum electrodynamical framework. A mechanism of similar nature can operate in molecular fluorescence, as we have shown in other recent work [2-4].

Section II gives a brief outline on the standard formulation that will be employed, including time-dependent perturbation theory and the Fermi rule. Sections III and IV detail how the stimulus beam affects one-photon and two-photon absorption, respectively; using typical values, calculations are made to determine the extent of absorption enhancement under various stimulus laser intensities. In Sec. V, the implications of differences in selection rules for the direct and indirectly influenced absorption processes are further considered and exemplified. The paper concludes with a discussion of potential applications, including quantum non-demolition measurements, in Sec. VI.

\section{QUANTUM FRAMEWORK}

The quantum electrodynamical analysis that follows is based on the Power-Zienau-Woolley formulation [5]. Within such a framework, the total nonrelativistic Hamiltonian, $H$, of a system containing a single molecule $A$ is expressed as

$$
H=H_{\mathrm{mol}}(A)+H_{\mathrm{int}}(A)+H_{\mathrm{rad}},
$$

where $H_{\text {mol }}(A)$ and $H_{\text {rad }}$ are the Hamiltonians for the molecule and radiation, respectively, and $H_{\text {int }}(A)$ represents the operator for the interaction between the radiation field and the molecule. The eigenstates, $|\psi\rangle$, of a basis Hamiltonian $H_{0}$ [equivalent 
to the right-hand side of Eq. (1) with $H_{\text {int }}$ excluded] form a composite set:

$$
|\psi\rangle=|\mathrm{mol}\rangle|\mathrm{rad}\rangle \equiv|A ; \mathrm{rad}\rangle .
$$

Here, $|\mathrm{mol}\rangle=|A\rangle$ defines the electronic state of the molecule, and $|\mathrm{rad}\rangle$ is the Fock (number) radiation state [6]. Within the electric-dipole approximation, and suppressing the implicit molecular dependences, $H_{\text {int }}(A)$ is defined by

$$
H_{\mathrm{int}}=-\boldsymbol{\mu} \cdot \mathbf{e}^{\perp}
$$

where the electric-dipole moment operator, $\boldsymbol{\mu}$, and the transverse electric field operator, $\mathbf{e}^{\perp}$, operate upon $|\mathrm{mol}\rangle$ and $|\mathrm{rad}\rangle$, respectively. Casting the electric field operator in terms of modes with photons of wave vector $\mathbf{k}$ and polarization $\eta$ we have the following mode expansion [7]:

$$
\begin{aligned}
\mathbf{e}^{\perp}= & i \sum_{\mathbf{k}, \eta}\left(\frac{\hbar c k}{2 \varepsilon_{0} V}\right)^{1 / 2}\left\{\mathbf{e}^{(\eta)}(\mathbf{k}) a^{(\eta)}(\mathbf{k}) e^{i\left(\mathbf{k} \cdot \mathbf{R}_{\xi}\right)}\right. \\
& \left.-\overline{\mathbf{e}}^{(\eta)}(\mathbf{k}) a^{\dagger(\eta)}(\mathbf{k}) e^{-i\left(\mathbf{k} \cdot \mathbf{R}_{\xi}\right)}\right\} .
\end{aligned}
$$

Here, $\mathbf{e}^{(\eta)}(\mathbf{k})$ is the polarization unit vector $\left[\overline{\mathbf{e}}^{(\eta)}(\mathbf{k})\right.$ being its complex conjugate], $V$ is an arbitrary quantization volume, $a^{(\eta)}(\mathbf{k})$ and $a^{\dagger(\eta)}(\mathbf{k})$ are, respectively, the photon annihilation and creation operators for the radiation mode $(\mathbf{k}, \eta)$. The latter operators act on radiation states through the relations: $a^{(\eta)}(\mathbf{k})|m(\mathbf{k}, \eta)\rangle=m^{1 / 2}|(m-1)(\mathbf{k}, \eta)\rangle$ and $a^{\dagger(\eta)}(\mathbf{k})|m(\mathbf{k}, \eta)\rangle=(m+1)^{1 / 2}|m+1(\mathbf{k}, \eta)\rangle$, the appearance of these operators in $H_{\text {int }}$ signifies a photon creation or annihilation. Furthermore, $e^{ \pm \mathrm{i}\left(\mathbf{k} \cdot \mathbf{R}_{\xi}\right)}$ denotes a position-dependent phase factor that plays no part in the observables for incoherent, single-center processes, and need not be considered further.

The quantum amplitude, $M_{F I}$, signifies a coupling between the initial system state $|I\rangle$ and final state $|F\rangle$, generally determined from time-dependent perturbation theory [8], giving

$$
\begin{aligned}
M_{F I}= & \sum_{p=0}^{\infty}\left\langle F\left|\left\{H_{\mathrm{int}}\left(T_{0} H_{\mathrm{int}}\right)^{p}\right\}\right| I\right\rangle \\
= & \langle F| H_{\mathrm{int}}+H_{\mathrm{int}} T_{0} H_{\mathrm{int}}+H_{\mathrm{int}} T_{0} H_{\mathrm{int}} T_{0} H_{\mathrm{int}} \\
& +H_{\mathrm{int}} T_{0} H_{\mathrm{int}} T_{0} H_{\mathrm{int}} T_{0} H_{\mathrm{int}}+\cdots|I\rangle \\
= & \left\langle F\left|H_{\mathrm{int}}\right| I\right\rangle+\sum_{R} \frac{\left\langle F\left|H_{\mathrm{int}}\right| R\right\rangle\left\langle R\left|H_{\mathrm{int}}\right| I\right\rangle}{\left(E_{I}-E_{R}\right)} \\
& +\sum_{R, S} \frac{\left\langle F\left|H_{\mathrm{int}}\right| S\right\rangle\left\langle S\left|H_{\mathrm{int}}\right| R\right\rangle\left\langle R\left|H_{\mathrm{int}}\right| I\right\rangle}{\left(E_{I}-E_{R}\right)\left(E_{I}-E_{S}\right)} \\
& +\sum_{R, S, T} \frac{\left\langle F\left|H_{\mathrm{int}}\right| T\right\rangle\left\langle T\left|H_{\mathrm{int}}\right| S\right\rangle\left\langle S\left|H_{\mathrm{int}}\right| R\right\rangle\left\langle R\left|H_{\mathrm{int}}\right| I\right\rangle}{\left(E_{I}-E_{R}\right)\left(E_{I}-E_{S}\right)\left(E_{I}-E_{T}\right)} \\
& +\cdots,
\end{aligned}
$$

where $T_{0} \approx\left(E_{I}-H_{0}\right)^{-1}$, in which $H_{0}$ is the unperturbed Hamiltonian that operates on the virtual intermediate states, denoted by $|R\rangle,|S\rangle,|T\rangle$, etc., and $E_{I}$ is the energy of system state $|I\rangle$. Moreover, each denominator term in Eq. (5) specifies a difference between the energies of the relevant system states (as denoted by the subscripts), and successive terms relate to processes of progressively higher order. The rate of any absorption process, $\Gamma$, is found from the Fermi rule [9],

$$
\Gamma=\frac{2 \pi \rho}{\hbar}\left|M_{F I}\right|^{2}
$$

where $\rho$ is a convolution of the density of radiation states and the density of final states for the absorber. This summary acts as a basis for the following derivation of the general expressions for laser-modified absorption, via the construction of statesequence diagrams [10].

\section{LASER-MODIFIED ONE-PHOTON ABSORPTION}

\section{A. Description of the mechanism}

One-photon absorption, arising from the application of a resonant laser beam, usually involves a single molecule-photon interaction (Fig. 1). This simple process is defined in terms of first-order time-dependent perturbation theory, i.e., the first term of line 2 or 3 of Eq. (5). In instances where throughput electromagnetic radiation (independent of the absorbing beam) is not present, higher-order terms are insignificant and they essentially denote self-energy corrections. However, subjecting the system to an off-resonant laser beam, specifically a stimulus with an optical frequency at which the molecule is transparent, this is no longer the case. Under such conditions, there is no net absorption or stimulated emission of the off-resonant beam, but elastic forward-scattering events do occur-photons are annihilated and created into the same radiation state (which thus emerges unchanged). These events engage through nonlinear coupling with the one-photon absorption; the net effect is to modify the transition dipole moment corresponding to light absorption. This mechanism entails three molecule-photon interactions, i.e., a third-order perturbation represented by the third term of Eq. (5). To be clear, this third term is always present for single-photon absorption, but it is usually insignificant compared to the first; however, the molecule-photon couplings become stronger on application of a sufficiently intense stimulus beam, resulting in the third-order term becoming important.

As a result of the quadratic dependence of absorption rates on the corresponding quantum amplitude, the rate equation for laser-modified one-photon $(1 \phi)$ absorption not only incorporates terms corresponding to first- and third-order

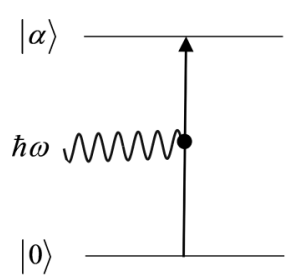

(a)

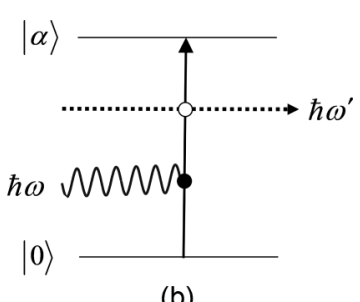

(b)
FIG. 1. Energy level representation for; (a) the linear interaction, and (b) the nonlinear interactions of laser-modified one-photon absorption. Molecular states are signified by horizontal lines with labels $|0\rangle$ and $|\alpha\rangle$ designating ground and excited states, respectively, the wavy line denotes an incoming resonant photon $(\hbar \omega)$ and the vertical arrow is a transition due to the resulting light absorption. The nonlinear case also involves the stimulus laser beam $\left(\hbar \omega^{\prime}\right)$ shown by the horizontal dashed arrow; the black and open dot symbolizes one and two molecule-photon interaction, respectively. 
perturbations, but also a cross term relating to a mixture of these perturbations, the latter term representing quantum interference. The net rate, determined from Eq. (6), is expressible as $\Gamma_{1 \phi} \sim\left|M_{F I}^{(1)}+M_{F I}^{(3)}\right|^{2}$, where $M_{F I}^{(1)}$ and $M_{F I}^{(3)}$ are quantum amplitudes for the first- and third-order interaction processes, respectively. Interference effects depend on the relative phases of the first- and third-order amplitudes; a similar phase will produce an enhanced absorption rate, dissimilar phases suggest suppression. The explicit forms of the relevant quantum amplitudes are determined by the insertion of Eqs. (3) and (4) into (5).

In the first-order interaction the only system states of significance are $|I\rangle=\left|A_{0} ; 1(\mathbf{k})\right\rangle$ and $|F\rangle=\left|A_{\alpha} ; 0(\mathbf{k})\right\rangle$, where the subscripts 0 and $\alpha$ denote the ground and excited states of molecule $A$, respectively-these two system states have an identical energy relating to $\hbar c k$ and, thus, satisfy conservation of energy. Assuming that $A$ is positioned at the origin, the expression $M_{F I}^{(1)}=\mathrm{i}\left(\hbar c k / 2 \varepsilon_{0} V\right)^{\frac{1}{2}} e_{i} \mu_{i}^{\alpha 0}$ is determined from the first term of Eq. (5); here, $\mathbf{e}$ is the polarization vector (the unit electric field vector) of the absorbed electromagnetic field, and both the shorthand notation $\boldsymbol{\mu}^{\alpha 0}=\langle\alpha|\boldsymbol{\mu}| 0\rangle$ and the convention of implied summation over Cartesian indexes (denoted by subscripts) are deployed.

The third-order (nonlinear) mechanism corresponds to $M_{F I}^{(3)}$, an expression for which invokes six distinct contributions, rather than just the one for $M_{F I}^{(1)}$. Each contribution involves a permutation in the time ordering of the three molecule-photon interactions, the detail of which provides the basis for constructing the molecular response tensor in the following subsection.

\section{B. Derivation of the molecular response tensor}

Following the analytical methodology described in other recent work [11], it is expedient to represent the interactions by unit vectors $\hat{\boldsymbol{i}}_{n}$ in a configuration space, and used in the set $I^{(3 ; 1 \phi)}$ as follows:

$$
I^{(3 ; 1 \phi)}=\left\{\hat{\boldsymbol{i}}_{1}, \hat{\boldsymbol{i}}_{2}, \hat{\boldsymbol{i}}_{3}\right\},
$$

where $\hat{\boldsymbol{i}}_{1}$ and $\hat{\boldsymbol{i}}_{2}$ are assigned to single-photon absorption and emission, respectively, in connection with the auxiliary beam; $\hat{\boldsymbol{i}}_{3}$ denotes resonant absorption of a photon (the sole interaction event of $\left.M_{F I}^{(1)}\right)$. Based on the number of occurrences, $C_{n}$, of each $\hat{\boldsymbol{i}}_{n}$ a binary system is constructed via the mapping

$$
\left(C_{1}, C_{2}, C_{3}\right) \rightarrow C_{1} C_{2} C_{3}=h,
$$

where $h$ is the corresponding hyperspace (decimal) index. The step number, $\kappa$, is found from

$$
\kappa=\sum_{n=1}^{3} C_{n} .
$$

From Eqs. (8) and (9), the variables $\kappa$ and $h$ are determined for each system state and reported in Table I. This tabulated information is used to construct an interaction network (Fig. 2): a plot of $h$ against $\kappa$, where the nodes represent the system states, and the connectors are only seen for cases when $\Delta h=$ 1,2 , and 4 , which relate to $\hat{\boldsymbol{i}}_{3}, \hat{\boldsymbol{i}}_{2}$, and $\hat{\boldsymbol{i}}_{1}$, respectively.
TABLE I. Step number $\kappa$, binary number $C_{1} C_{2} C_{3}$ and hyperspace number $h$ for each system state of laser-modified one-photon absorption (for the nonlinear, third-order interaction). This information is used to construct Figs. 2 and 3.

\begin{tabular}{lcc}
\hline$\kappa$ & $C_{1} C_{2} C_{3}$ & $h$ \\
\hline 0 & 000 & 0 \\
1 & 001 & 1 \\
1 & 010 & 2 \\
1 & 100 & 4 \\
2 & 011 & 3 \\
2 & 101 & 5 \\
2 & 110 & 6 \\
3 & 111 & 7 \\
\hline \hline
\end{tabular}

Using a shortcut procedure to find the hyperpolarizability part of $M_{F I}^{(3)}$, an adjacency matrix [12] method is now applied. This first involves construction of a connectivity matrix $M_{C}$, which acts as a molecular state projection of $H_{i n t}$ and produces the required numerator. This matrix, expressed in a projection form, is written as

$$
\begin{aligned}
M_{C}= & |0\rangle \hat{\boldsymbol{i}}_{3}\langle 1|+| 0\rangle \hat{\boldsymbol{i}}_{2}\langle 2|+| 0\rangle \hat{\boldsymbol{i}}_{1}\langle 4|+| 1\rangle \hat{\boldsymbol{i}}_{2}\langle 3|+| 1\rangle \hat{\boldsymbol{i}}_{1}\langle 5| \\
& +|2\rangle \hat{\boldsymbol{i}}_{3}\langle 3|+| 2\rangle \hat{\boldsymbol{i}}_{1}\langle 6|+| 3\rangle \hat{\boldsymbol{i}}_{1}\langle 7|+| 4\rangle \hat{\boldsymbol{i}}_{3}\langle 5| \\
& +|4\rangle \hat{\boldsymbol{i}}_{2}\langle 6|+| 5\rangle \hat{\boldsymbol{i}}_{2}\langle 7|+| 6\rangle \hat{\boldsymbol{i}}_{3}\langle 7|
\end{aligned}
$$

representing all the possible single (forward) steps, i.e., $\Delta \kappa=1$, between nodes in Fig. 2. To identify the various routes from the initial $|0\rangle$ to the final $|7\rangle$ state requires cubing the right-hand side of Eq. (10), since this is a three-interaction process, so that

$$
\begin{aligned}
M_{C}^{3}= & \hat{\boldsymbol{i}}_{3} \hat{\boldsymbol{i}}_{2} \hat{\boldsymbol{i}}_{1}+\hat{\boldsymbol{i}}_{3} \hat{\boldsymbol{i}}_{1} \hat{\boldsymbol{i}}_{2}+\hat{\boldsymbol{i}}_{2} \hat{\boldsymbol{i}}_{3} \hat{\boldsymbol{i}}_{1}+\hat{\boldsymbol{i}}_{2} \hat{\boldsymbol{i}}_{1} \hat{\boldsymbol{i}}_{3}+\hat{\boldsymbol{i}}_{1} \hat{\boldsymbol{i}}_{3} \hat{\boldsymbol{i}}_{2}+\hat{\boldsymbol{i}}_{1} \hat{\boldsymbol{i}}_{2} \hat{\boldsymbol{i}}_{3} \\
= & \mu_{k}^{r 0} \mu_{j}^{s r} \mu_{i}^{\alpha s}+\mu_{k}^{r 0} \mu_{i}^{s r} \mu_{j}^{\alpha s}+\mu_{j}^{r 0} \mu_{k}^{s r} \mu_{i}^{\alpha s} \\
& +\mu_{j}^{r 0} \mu_{i}^{s r} \mu_{k}^{\alpha s}+\mu_{i}^{r 0} \mu_{k}^{s r} \mu_{j}^{\alpha s}+\mu_{i}^{r 0} \mu_{j}^{s r} \mu_{k}^{\alpha s}
\end{aligned}
$$

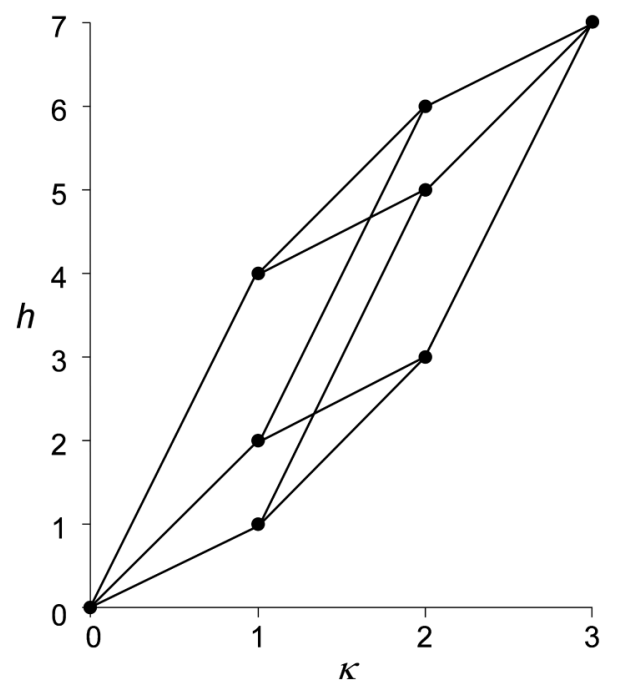

FIG. 2. The three-interaction plane network depicting lasermodified one-photon absorption. The integer step number, $\kappa$, is plotted against the integer hyperspace number, $h$. 
TABLE II. All system states and their associated energies, $E_{r}$, determined for Fig. 3. Here, $\left|r_{\kappa}^{h}\right\rangle$ represents a state denoted by the step number $\kappa$ and hyperspace number $h$, and $\left|A_{\kappa} ; n_{\omega}, n_{\omega^{\prime}}\right\rangle$ is the system state decomposed into molecular states, $A_{\kappa}$, and the relevant radiation states.

\begin{tabular}{lcc}
\hline \hline System state $\left|r_{\kappa}^{h}\right\rangle$ & $\left|A_{\kappa} ; n_{\omega}, n_{\omega^{\prime}}\right\rangle$ & Energy $E_{r}$ \\
\hline$|I\rangle \equiv\left|r_{0}^{0}\right\rangle$ & $\left|A_{0} ; 1,1\right\rangle$ & $E_{0}+\hbar\left(\omega+\omega^{\prime}\right)$ \\
$\left|r_{1}^{1}\right\rangle$ & $\left|A_{1} ; 0,1\right\rangle$ & $E_{r}+\hbar \omega^{\prime}$ \\
$\left|r_{1}^{2}\right\rangle$ & $\left|A_{1} ; 1,2\right\rangle$ & $E_{r}+\hbar\left(\omega+2 \omega^{\prime}\right)$ \\
$\left|r_{1}^{4}\right\rangle$ & $\left|A_{1} ; 1,0\right\rangle$ & $E_{r}+\hbar \omega$ \\
$\left|r_{2}^{3}\right\rangle$ & $\left|A_{2} ; 0,2\right\rangle$ & $E_{s}+2 \hbar \omega^{\prime}$ \\
$\left|r_{2}^{5}\right\rangle$ & $\left|A_{2} ; 0,0\right\rangle$ & $E_{s}$ \\
$\left|r_{2}^{6}\right\rangle$ & $\left|A_{2} ; 1,1\right\rangle$ & $E_{s}+\hbar\left(\omega+\omega^{\prime}\right)$ \\
$|F\rangle \equiv\left|r_{3}^{7}\right\rangle$ & $\left|A_{3} ; 0,1\right\rangle$ & $E_{\alpha}+\hbar \omega^{\prime}$ \\
\hline \hline
\end{tabular}

where $\hat{\boldsymbol{i}}_{3}$ relates to the transition dipole moment $\mu_{i}$ (the subscript $i$ denotes resonant absorption, as $M_{F I}^{(1)}$ ), $\hat{i}_{2}$ corresponds to $\mu_{j}$, and $\hat{\boldsymbol{i}}_{1}$ to $\mu_{k}$. Moreover, the subscripts $r$ and $s$ denote molecular virtual intermediate states, which are simply recasts of $\kappa=1$ and 2, respectively. Equation (11) also includes the time orderings of the molecule-photon interactions (signified by the superscripts $r 0, s r$, then $\alpha s$ ), and the six terms represent the six routes from left to right in Fig. 2. To ascertain the energy denominator for each term, consistent with Eq. (5), it is necessary to derive a further matrix that represents the system energetics. This is generally given by

$$
M_{E}=\sum_{h=1}^{h_{\max }-1}|h\rangle S_{h}\langle h|,
$$

where $S_{h}=\left(E_{0}-E_{r_{k}^{h}}\right)^{-1}$, i.e., an eigenvalue of $T_{0}$, is a factor determined from the energy associated with the relevant system states as given by Table II.

The next stage in the determination of the hyperpolarizability tensor is to use the sequence $M_{C} M_{E} M_{C} M_{E} M_{C}$. This includes the three steps from the initial to the final state, i.e., $M_{C}^{3}$ of Eq. (11), and the energetics information of the relevant node that follows each step, held within the matrix $M_{E}$ as defined by Eq. (12). On multiplication of the matrices in the above sequence, an order congruent to the third term of the second or third line of (5), Eq. (11) delivers a specific form of hyperpolarizability, $\beta_{i j k}^{\alpha 0}\left(\omega^{\prime}\right)$, signifying the molecular response term in $M_{F I}^{(3)}$ :

$$
\begin{aligned}
\beta_{i j k}^{\alpha 0}\left(\omega^{\prime}\right)= & \hat{\boldsymbol{i}}_{3} S_{1} \hat{\boldsymbol{i}}_{2} S_{3} \hat{\boldsymbol{i}}_{1}+\hat{\boldsymbol{i}}_{3} S_{1} \hat{\boldsymbol{i}}_{1} S_{5} \hat{\boldsymbol{i}}_{2}+\hat{\boldsymbol{i}}_{2} S_{2} \hat{\boldsymbol{i}}_{3} S_{3} \hat{\boldsymbol{i}}_{1} \\
& +\hat{\boldsymbol{i}}_{2} S_{2} \hat{\boldsymbol{i}}_{1} S_{6} \hat{\boldsymbol{i}}_{3}+\hat{\boldsymbol{i}}_{1} S_{4} \hat{\boldsymbol{i}}_{3} S_{5} \hat{\boldsymbol{i}}_{2}+\hat{\boldsymbol{i}}_{1} S_{4} \hat{\boldsymbol{i}}_{2} S_{6} \hat{\boldsymbol{i}}_{3} \\
= & \sum_{r, s \neq 0}\left(\frac{\mu_{i}^{\alpha s} \mu_{j}^{s r} \mu_{k}^{r 0}}{\tilde{E}_{s 0}\left(\tilde{E}_{r 0}-\hbar \omega^{\prime}\right)}+\frac{\mu_{i}^{\alpha s} \mu_{k}^{s r} \mu_{j}^{r 0}}{\tilde{E}_{s 0}\left(\tilde{E}_{r 0}+\hbar \omega^{\prime}\right)}\right) \\
& +\sum_{r, s}\left(\frac{\mu_{j}^{\alpha s} \mu_{i}^{s r} \mu_{k}^{r 0}}{\left(\tilde{E}_{r 0}-\hbar \omega^{\prime}\right)\left(\tilde{E}_{s \alpha}-\hbar \omega^{\prime}\right)}\right.
\end{aligned}
$$

$$
\begin{aligned}
& \left.+\frac{\mu_{k}^{\alpha s} \mu_{i}^{s r} \mu_{j}^{r 0}}{\left(\tilde{E}_{r 0}+\hbar \omega^{\prime}\right)\left(\tilde{E}_{s \alpha}+\hbar \omega^{\prime}\right)}\right) \\
& +\sum_{r \neq \alpha, s}\left(\frac{\mu_{j}^{\alpha s} \mu_{k}^{s r} \mu_{i}^{r 0}}{\tilde{E}_{r \alpha}\left(\tilde{E}_{s \alpha}-\hbar \omega^{\prime}\right)}+\frac{\mu_{k}^{\alpha s} \mu_{j}^{s r} \mu_{i}^{r 0}}{\tilde{E}_{r \alpha}\left(\tilde{E}_{s \alpha}+\hbar \omega^{\prime}\right)}\right)
\end{aligned}
$$

Here, $E_{x y}=E_{x}-E_{y}$ is an energy difference between two states (for example, $E_{\alpha 0} \equiv \hbar \omega$ ) and the excluded summands are due to the rules of perturbation theory. Moreover, the tildes act as a reminder to add to the excited state energies, in the case of near-resonance conditions, imaginary terms to accommodate damping: explicitly $\tilde{E}_{x y}=E_{x y}-\frac{1}{2} \mathrm{i} \hbar \gamma$, where $\gamma$ represents the full width at half maximum linewidth near-resonance [13]. To clarify, Eq. (13) is a transition hyperpolarizability tensor (not to be confused with the elastic second-harmonic scattering hyperpolarizability involved in frequency doubling). The present tensor quantifies the crux of the single-photon analysis, representing the nonlinear engagement of the molecule with the throughput stimulus radiation in the course of its excitation.

\section{Rate expression for laser-modified absorption}

Since the premultiplier of $M_{F I}^{(3)}$ is independent of the time orderings, the molecule-photon interactions of two annihilations and one creation produce $i m k^{\prime} k^{1 / 2}\left(\hbar c / 2 \varepsilon_{0} V\right)^{3 / 2} e_{i} e_{j}^{\prime} \bar{e}_{k}^{\prime}$ from Eq. (4), assuming that $m \approx m+1$, so that

$$
M_{F I}^{(3)}=i m k^{\prime} k^{\frac{1}{2}}\left(\frac{\hbar c}{2 \varepsilon_{0} V}\right)^{3 / 2} e_{i} e_{j}^{\prime} \bar{e}_{k}^{\prime} \beta_{i j k}^{\alpha 0}\left(\omega^{\prime}\right),
$$

where the energy $\hbar c k^{\prime} \equiv \hbar \omega^{\prime}$ and $\mathbf{e}^{\prime}$ denotes the polarization vector of the throughput beam. A visual aid for describing the nonlinear component of laser-modified one-photon absorption is the state-sequence diagram of Fig. 3, constructed from Fig. 2 and Table II. The six distinct contributions are identified by the six different pathways from the initial to the final state in Fig. 3. Through employment of Eq. (6) with $M_{F I}^{(1)}$ and $M_{F I}^{(3)}$, the rate

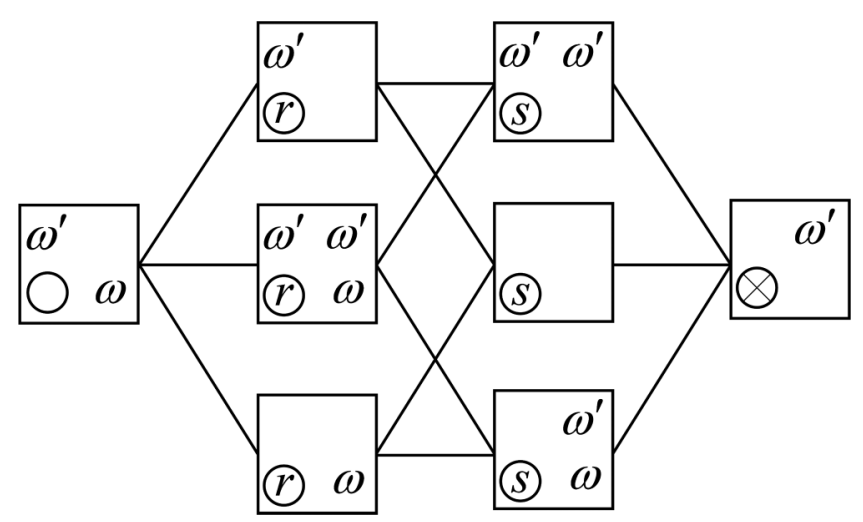

FIG. 3. State-sequence diagram for the nonlinear interaction of laser-modified one-photon absorption. A photon from mode $\omega^{\prime}$ is created and photon modes $\omega$ and $\omega^{\prime}$ are annihilated. The initial state is on the left-hand side, and the final state on the right; each route between the two signifies a different quantum pathway. The crossed circle represents the molecule in an excited state, lettered circles an intermediate (virtual) state, and an unfilled circle a ground state. 


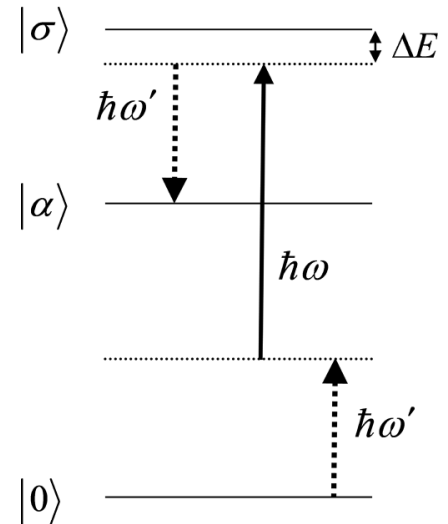

FIG. 4. Energy level scheme for a three-level system. As Fig. 1, but includes dotted horizontal lines that represent virtual states and $|\sigma\rangle$ as a higher electronic state, which is approximately $\hbar \omega^{\prime}$ above $|\alpha\rangle$ (the difference between which is given by $\Delta E$ ). Dashed vertical arrows are transitions due to the stimulus laser beam.

for laser-modified one-photon absorption is finally determined as

$$
\begin{aligned}
\Gamma_{1 \phi}= & \left(\frac{\pi \rho I}{c \hbar \varepsilon_{0}}\right) e_{i} \bar{e}_{j}\left[\mu_{i}^{(0) \alpha 0} \bar{\mu}_{j}^{(0) \alpha 0}+\left(I^{\prime} / c \varepsilon_{0}\right) \mu_{i}^{(0) \alpha 0} \bar{\mu}_{j}^{(2) \alpha 0}\left(\omega^{\prime}\right)\right. \\
& \left.+\left(I^{\prime 2} / 4 c^{2} \varepsilon_{0}^{2}\right) \mu_{i}^{(2) \alpha 0}\left(\omega^{\prime}\right) \bar{\mu}_{j}^{(2) \alpha 0}\left(\omega^{\prime}\right)\right]
\end{aligned}
$$

where $I=\hbar c^{2} k / V$ is the irradiance of the absorbing (resonant) beam, $I^{\prime}=m \hbar c^{2} k^{\prime} / V$ is the throughput laser irradiance, and the overbar on a polarization component allows for the possibility of complex (such as circular) polarization. Equation (15) is interpreted as one-photon absorption associated with an effective excitation dipole whose zeroth-order contribution, independent of the stimulus beam, is designated $\mu_{i}^{(0) \alpha 0} \equiv \mu_{i}^{\alpha 0}$, and the contribution quadratic in the electric field of the stimulus beam is $\mu_{i}^{(2) \alpha 0}\left(\omega^{\prime}\right) \equiv e_{j}^{\prime} \bar{e}_{k}^{\prime} \beta_{i j k}^{\alpha 0}\left(\omega^{\prime}\right)$. The former represents direct resonant absorption, and the latter a nonlinear correction term that couples with the off-resonant radiation of frequency $\omega^{\prime}$ (corresponding to elastic forward scattering). The stimulus beam thus effects a modification of the excitation dipole moment through $\boldsymbol{\mu}^{(2) \alpha 0}\left(\omega^{\prime}\right)$.

The initial term on the right-hand side in Eq. (15) corresponds to resonant absorption, independent of the stimulus beam. The third term signifies a coupling of the elastically forward-scattered stimulus beam with the one-photon absorption. However it is the second term (linear in $I^{\prime}$ ), signifying a quantum interference of these two amplitudes, that represents the leading correction; this is the focus of our analysis in the following subsection addressing a three-level system.

\section{Three-level system}

To achieve numerical estimates, and to identify specific conditions for rate enhancement or suppression, a system is now considered in which the molecule has just three energy levels significantly involved in the interaction (Fig. 4)-a physically reasonable assumption that will introduce simplifications to the general expression of Eq. (15). In addition, for extra calculational ease, we assume that the stimulus beam is linearly polarized and relevant dipoles and polarization vectors are broadly parallel to each other. The virtue of this supposition is that it is the structure of the energy denominators in Eq. (13) that primarily dictates the degree of enhancement or suppression of the one-photon absorption rate. The magnitude of such denominators is determined by the relative positioning of the molecular energy levels relative to the photon energy of the stimulus beam.

In detail, we utilize a three-level molecule with optically prominent states $|0\rangle,|\sigma\rangle$, and $|\alpha\rangle$, where the latter is the lowest electronic excited state. Moreover, it is assumed that the stimulus light delivers an optical frequency $\omega^{\prime}$ with $\hbar \omega^{\prime}<E_{\alpha 0}$, excluding direct excitation from the ground state to $|\sigma\rangle$. It follows from Eq. (13) that the maximum effect is achievable for cases where $|\sigma\rangle$ has an energy approximately $\hbar \omega^{\prime}$ above $|\alpha\rangle$, so that $\Delta \tilde{E}=\tilde{E}_{\sigma \alpha}-\hbar \omega^{\prime}$. Effecting the sums over intermediate states $r$ and $s$ in Eq. (13), the candidacy for these intermediate states is now limited to $0, \alpha$, or $\sigma$ (except where excluded by the restrictions on summation), and it is the third term on the second line that yields the smallest magnitude denominator. Thus, on setting $r=0$ and $s=\sigma$ we have

$$
e_{i} \mu_{i}^{(2) \alpha 0}\left(\omega^{\prime}\right) \approx-\frac{\mu^{3}}{\hbar \omega^{\prime} \Delta \tilde{E}} .
$$

Broadly equivalent magnitudes are assumed for the relevant transition dipoles (signified by $\boldsymbol{\mu} \equiv \mathbf{e} \cdot \boldsymbol{\mu}^{(0) \alpha 0}$ ).

On analysis of Eq. (16) it emerges that, for $E_{\sigma \alpha}<\hbar \omega^{\prime}$, the sign of the leading correction will be positive, signifying that the stimulus beam enhances the one-photon absorption rate; note that the factor $I^{\prime} / c \varepsilon_{0}$ within Eq. (15) is always positive. In contrast, the sign is negative for $E_{\sigma \alpha}>\hbar \omega^{\prime}$, representing suppressed absorption. Typical values for $\Gamma_{1 \phi}$ may be calculated for various stimulus laser intensities; for example, setting $\mu=15 \times 10^{-30} \mathrm{C} \mathrm{m}, \Delta E=-10^{-20} \mathrm{~J}$, and $\hbar \omega^{\prime}=10^{19} \mathrm{~J}$, the rate of one-photon absorption proves to be enhanced by $\sim 20 \%$ for an irradiance of $2 \times 10^{15} \mathrm{~W} \mathrm{~m}^{-2}$. Even at a level of $10^{14} \mathrm{~W} \mathrm{~m}^{-2}$, it can be anticipated that the resulting change in absorption rate will be readily measurable by the use of modulation detection techniques [14].

\section{LASER-MODIFIED TWO-PHOTON ABSORPTION}

\section{A. Description of the mechanism}

The absorption of two identical photons by a molecule, via interaction with a resonant laser beam, is primarily considered a second-order perturbation. On irradiating the system with a stimulus beam (of sufficient intensity) the rate of absorption will again be affected. This process entails four moleculephoton interactions, namely a fourth-order perturbation accordingly represented by the fourth term of Eq. (5). Proceeding in a directly analogous way to that described in Sec. III, the rate expression for laser-modified two-photon $(2 \phi)$ absorption emerges in a form expressible as $\Gamma_{2 \phi} \sim\left|M_{F I}^{(2)}+M_{F I}^{(4)}\right|^{2}$, where $M_{F I}^{(2)}$ and $M_{F I}^{(4)}$ are quantum amplitudes for the second- and fourth-order interaction processes, respectively. The explicit form of the relevant quantum amplitudes are determined by the insertion of Eqs. (3) and (4) into (5).

Laser-modified two-photon absorption entails two identical interactions; that is, the annihilated photons from the input 


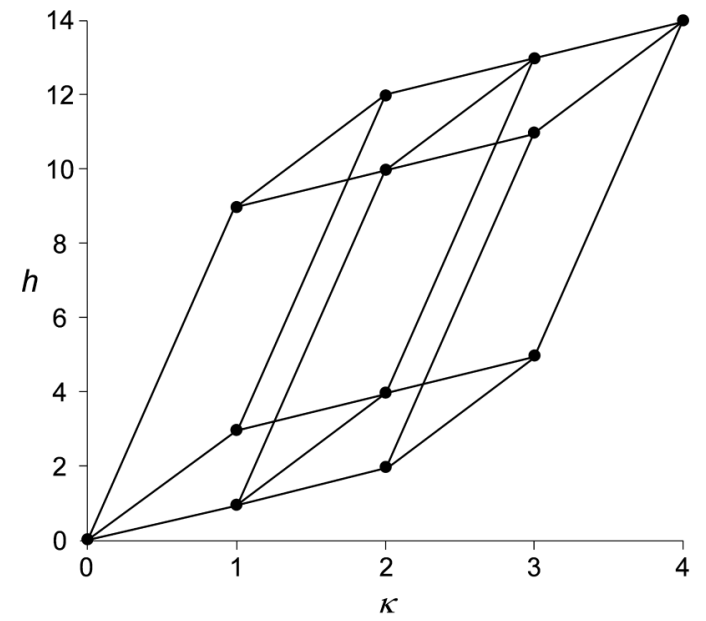

FIG. 5. The four-interaction plane network depicting lasermodified two-photon absorption.

resonant beam are interchangeable. As a result of such degeneracy, the number of distinct interactions is less than the total. Therefore, in constructing the configuration space representation each unit vector $\hat{\boldsymbol{i}}_{n}$ now requires the assignment of a coefficient, which denotes the number of occurrences of each interaction. On employment of this coefficient, the second- and fourth-order mechanisms are defined by the set $I^{(2 ; 2 \phi)}=\left\{2 \hat{\boldsymbol{i}}_{3}\right\}$ and $I^{(4 ; 2 \phi)}=\left\{\hat{\boldsymbol{i}}_{1}, \hat{\boldsymbol{i}}_{2}, 2 \hat{\boldsymbol{i}}_{3}\right\}$, respectively.

In the second-order mechanism, the initial and final system states are denoted by $|I\rangle=\left|A_{0} ; 2(\mathbf{k})\right\rangle$ and $|F\rangle=\left|A_{\alpha^{\prime}} ; 0(\mathbf{k})\right\rangle$, where $\alpha^{\prime}$ represents a two-photon excited state of molecule $A$; the intermediate state is expressed as $\left|r_{1}^{1}\right\rangle=\left|A_{r} ; 1(\mathbf{k})\right\rangle$. Using the earlier procedure, the following is found:

$$
M_{F I}^{(2)}=\frac{\hbar c k}{2^{1 / 2} \varepsilon_{0} V} e_{i} e_{j} \alpha_{i j}^{\alpha^{\prime} 0},
$$

where the two-photon absorption or transition polarizability tensor $\alpha_{i j}^{\alpha^{\prime} 0}$ is given by

$$
\alpha_{i j}^{\alpha^{\prime} 0}=\hat{\boldsymbol{i}}_{3} S_{1} \hat{\boldsymbol{i}}_{3}=\frac{1}{2} \sum_{r}\left(\frac{\mu_{i}^{\alpha^{\prime} r} \mu_{j}^{r 0}+\mu_{j}^{\alpha^{\prime} r} \mu_{i}^{r 0}}{\tilde{E}_{r 0}-\hbar \omega}\right) .
$$

Here, the interaction $\hat{\boldsymbol{i}}_{3}$ is assigned to $\mu_{i}$ and $\mu_{j}$ for the respective first and second instance that it occurs, and the premultiplier is employed to omit double counting. Equation (18) is $i, j$-index symmetric since it represents two identical molecule-photon interactions.

For the more intricate fourth-order mechanism, promoted by engaging the stimulus beam, an interaction network (Fig. 5) and state-sequence diagram (Fig. 6) are constructed. These diagrams again originate from the number of occurrences of each interaction though, since two of the interactions are identical, the coordinate system is now based on ternary numerals (Table III).

\section{B. Derivation of the molecular response tensor}

Using the shortcut procedure to find the second-order hyperpolarizability part of $M_{F I}^{(4)}$, as discussed in the counterpart single-photon case, the connectivity matrix is here

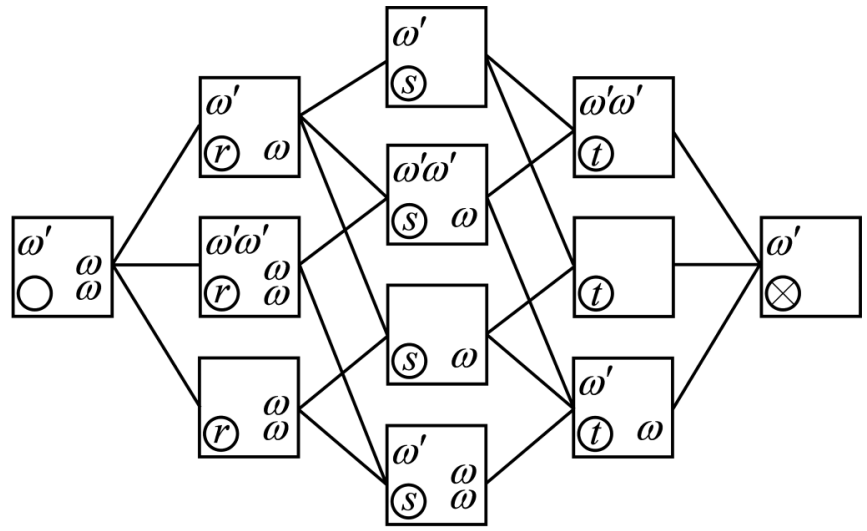

FIG. 6. State-sequence diagram for the stimulus beam dependent part of laser-modified two-photon absorption. As Fig. 3, but an additional photon is annihilated from mode $\omega$.

written as

$$
\begin{aligned}
M_{C}= & |0\rangle \hat{\boldsymbol{i}}_{3}\langle 1|+| 0\rangle \hat{\boldsymbol{i}}_{2}\langle 3|+| 0\rangle \hat{\boldsymbol{i}}_{1}\langle 9|+| 1\rangle \hat{\boldsymbol{i}}_{3}\langle 2|+| 1\rangle \hat{\boldsymbol{i}}_{2}\langle 4| \\
& +|1\rangle \hat{\boldsymbol{i}}_{1}\langle 10|+| 3\rangle \hat{\boldsymbol{i}}_{3}\langle 4|+| 3\rangle \hat{\boldsymbol{i}}_{1}\langle 12|+| 2\rangle \hat{\boldsymbol{i}}_{2}\langle 5|+| 2\rangle \hat{\boldsymbol{i}}_{1}\langle 11| \\
& +|4\rangle \hat{\boldsymbol{i}}_{3}\langle 5|+| 4\rangle \hat{\boldsymbol{i}}_{1}\langle 13|+| 5\rangle \hat{\boldsymbol{i}}_{1}\langle 14|+| 9\rangle \hat{\boldsymbol{i}}_{3}\langle 10| \\
& +|9\rangle \hat{\boldsymbol{i}}_{2}\langle 12|+| 10\rangle \hat{\boldsymbol{i}}_{3}\langle 11|+| 10\rangle \hat{\boldsymbol{i}}_{2}\langle 13|+| 12\rangle \hat{\boldsymbol{i}}_{3}\langle 13| \\
& +|11\rangle \hat{\boldsymbol{i}}_{2}\langle 14|+| 13\rangle \hat{\boldsymbol{i}}_{3}\langle 14|,
\end{aligned}
$$

which represents all the possible forward steps between nodes in Fig. 5. To identify the various routes from $|0\rangle$ to $|14\rangle$ requires that Eq. (19) is quadrupled, since it is a four-interaction process. With inclusion of $M_{E}$, employed to ascertain the energy denominators for each term (from Table IV), in between the $M_{C}$ factors, i.e.,

$$
\begin{aligned}
& M_{C} M_{E} M_{C} M_{E} M_{C} M_{E} M_{C} \\
& =\hat{\boldsymbol{i}}_{3} S_{1} \hat{\boldsymbol{i}}_{3} S_{2} \hat{\boldsymbol{i}}_{2} S_{5} \hat{\boldsymbol{i}}_{1}+\hat{\boldsymbol{i}}_{3} S_{1} \hat{\boldsymbol{i}}_{3} S_{2} \hat{\boldsymbol{i}}_{1} S_{11} \hat{\boldsymbol{i}}_{2}+\hat{\boldsymbol{i}}_{3} S_{1} \hat{\boldsymbol{i}}_{2} S_{4} \hat{\boldsymbol{i}}_{1} S_{13} \hat{\boldsymbol{i}}_{3} \\
& \quad+\hat{\boldsymbol{i}}_{3} S_{1} \hat{\boldsymbol{i}}_{2} S_{4} \hat{\boldsymbol{i}}_{3} S_{5} \hat{\boldsymbol{i}}_{1}+\hat{\boldsymbol{i}}_{3} S_{1} \hat{\boldsymbol{i}}_{1} S_{10} \hat{\boldsymbol{i}}_{3} S_{11} \hat{\boldsymbol{i}}_{2}+\hat{\boldsymbol{i}}_{3} S_{1} \hat{\boldsymbol{i}}_{1} S_{10} \hat{\boldsymbol{i}}_{2} S_{13} \hat{\boldsymbol{i}}_{3} \\
& \quad+\hat{\boldsymbol{i}}_{2} S_{3} \hat{\boldsymbol{i}}_{3} S_{4} \hat{\boldsymbol{i}}_{3} S_{5} \hat{\boldsymbol{i}}_{1}+\hat{\boldsymbol{i}}_{2} S_{3} \hat{\boldsymbol{i}}_{3} S_{4} \hat{\boldsymbol{i}}_{1} S_{13} \hat{\boldsymbol{i}}_{3}+\hat{\boldsymbol{i}}_{2} S_{3} \hat{\boldsymbol{i}}_{1} S_{12} \hat{\boldsymbol{i}}_{3} S_{13} \hat{\boldsymbol{i}}_{3} \\
& \quad+\hat{\boldsymbol{i}}_{1} S_{9} \hat{\boldsymbol{i}}_{2} S_{12} \hat{\boldsymbol{i}}_{3} S_{13} \hat{\boldsymbol{i}}_{3}+\hat{\boldsymbol{i}}_{1} S_{9} \hat{\boldsymbol{i}}_{3} S_{10} \hat{\boldsymbol{i}}_{3} S_{11} \hat{\boldsymbol{i}}_{2}+\hat{\boldsymbol{i}}_{1} S_{9} \hat{\boldsymbol{i}}_{3} S_{10} \hat{\boldsymbol{i}}_{2} S_{13} \hat{\boldsymbol{i}}_{3},
\end{aligned}
$$

TABLE III. As Table I, but for laser-modified two-photon absorption; here, $C_{1} C_{2} C_{3}$ is a ternary number. This information is used to construct Figs. 5 and 6.

\begin{tabular}{lcc}
\hline \hline$\kappa$ & $C_{1} C_{2} C_{3}$ & $h$ \\
\hline 0 & 000 & 0 \\
1 & 001 & 1 \\
1 & 010 & 3 \\
1 & 100 & 9 \\
2 & 002 & 2 \\
2 & 011 & 4 \\
2 & 101 & 10 \\
2 & 110 & 12 \\
3 & 012 & 5 \\
3 & 102 & 11 \\
3 & 111 & 13 \\
4 & 112 & 14 \\
\hline \hline
\end{tabular}


the second-order transition hyperpolarizability, $\chi_{i j k l}^{\alpha^{\prime} 0}\left(\omega^{\prime}\right)$, is determined as

$$
\begin{aligned}
& \chi_{i j k l}^{\alpha^{\prime} 0}\left(\omega^{\prime}\right)=\sum_{r, t, s \neq \alpha^{\prime}}\left(\frac{\mu_{l}^{\alpha^{\prime} t} \mu_{k}^{t s} \mu_{j}^{s r} \mu_{i}^{r 0}}{\tilde{E}_{s \alpha^{\prime}}\left(\tilde{E}_{r 0}-\frac{1}{2} \tilde{E}_{\alpha^{\prime} 0}\right)\left(\tilde{E}_{t \alpha^{\prime}}+\hbar \omega^{\prime}\right)}+\frac{\mu_{k}^{\alpha^{\prime} t} \mu_{l}^{t s} \mu_{j}^{s r} \mu_{i}^{r 0}}{\tilde{E}_{s \alpha^{\prime}}\left(\tilde{E}_{r 0}-\frac{1}{2} \tilde{E}_{\alpha^{\prime} 0}\right)\left(\tilde{E}_{t \alpha^{\prime}}-\hbar \omega^{\prime}\right)}\right) \\
& +\sum_{r, s \neq 0, t}\left(\frac{\mu_{j}^{\alpha^{\prime} t} \mu_{i}^{t s} \mu_{l}^{s r} \mu_{k}^{r 0}}{\tilde{E}_{s 0}\left(\tilde{E}_{r 0}+\hbar \omega^{\prime}\right)\left(\tilde{E}_{t 0}-\frac{1}{2} \tilde{E}_{\alpha^{\prime} 0}\right)}+\frac{\mu_{j}^{\alpha^{\prime} t} \mu_{i}^{t s} \mu_{k}^{s r} \mu_{l}^{r 0}}{\tilde{E}_{s 0}\left(\tilde{E}_{r 0}-\hbar \omega^{\prime}\right)\left(\tilde{E}_{t 0}-\frac{1}{2} \tilde{E}_{\alpha^{\prime} 0}\right)}\right) \\
& +\sum_{r, s, t}\left(\frac{\mu_{j}^{\alpha^{\prime} t} \mu_{l}^{t s} \mu_{k}^{s r} \mu_{i}^{r 0}}{\left(\tilde{E}_{r 0}-\frac{1}{2} \tilde{E}_{\alpha^{\prime} 0}\right)\left(\tilde{E}_{s 0}-\frac{1}{2} \tilde{E}_{\alpha^{\prime} 0}+\hbar \omega^{\prime}\right)\left(\tilde{E}_{t 0}-\frac{1}{2} \tilde{E}_{\alpha^{\prime} 0}\right)}+\frac{\mu_{l}^{\alpha^{\prime} t} \mu_{j}^{t s} \mu_{k}^{s r} \mu_{i}^{r 0}}{\left(\tilde{E}_{r 0}-\frac{1}{2} \tilde{E}_{\alpha^{\prime} 0}\right)\left(\tilde{E}_{s 0}-\frac{1}{2} \tilde{E}_{\alpha^{\prime} 0}+\hbar \omega^{\prime}\right)\left(\tilde{E}_{t \alpha^{\prime}}+\hbar \omega^{\prime}\right)}\right. \\
& +\frac{\mu_{k}^{\alpha^{\prime} t} \mu_{j}^{t s} \mu_{l}^{s r} \mu_{i}^{r 0}}{\left(\tilde{E}_{r 0}-\frac{1}{2} \tilde{E}_{\alpha^{\prime} 0}\right)\left(\tilde{E}_{s 0}-\frac{1}{2} \tilde{E}_{\alpha^{\prime} 0}-\hbar \omega^{\prime}\right)\left(\tilde{E}_{t \alpha^{\prime}}-\hbar \omega^{\prime}\right)}+\frac{\mu_{j}^{\alpha^{\prime} t} \mu_{k}^{t s} \mu_{l}^{s r} \mu_{i}^{r 0}}{\left(\tilde{E}_{r 0}-\frac{1}{2} \tilde{E}_{\alpha^{\prime} 0}\right)\left(\tilde{E}_{s 0}-\frac{1}{2} \tilde{E}_{\alpha^{\prime} 0}-\hbar \omega^{\prime}\right)\left(\tilde{E}_{t 0}-\frac{1}{2} \tilde{E}_{\alpha^{\prime} 0}\right)} \\
& +\frac{\mu_{l}^{\alpha^{\prime} t} \mu_{j}^{t s} \mu_{i}^{s r} \mu_{k}^{r 0}}{\left(\tilde{E}_{r 0}+\hbar \omega^{\prime}\right)\left(\tilde{E}_{s 0}-\frac{1}{2} \tilde{E}_{\alpha^{\prime} 0}+\hbar \omega^{\prime}\right)\left(\tilde{E}_{t \alpha^{\prime}}+\hbar \omega^{\prime}\right)}+\frac{\mu_{j}^{\alpha^{\prime} t} \mu_{l}^{t s} \mu_{i}^{s r} \mu_{k}^{r 0}}{\left(\tilde{E}_{r 0}+\hbar \omega^{\prime}\right)\left(\tilde{E}_{s 0}-\frac{1}{2} \tilde{E}_{\alpha^{\prime} 0}+\hbar \omega^{\prime}\right)\left(\tilde{E}_{t 0}-\frac{1}{2} \tilde{E}_{\alpha^{\prime} 0}\right)} \\
& \left.+\frac{\mu_{k}^{\alpha^{\prime} t} \mu_{j}^{t s} \mu_{i}^{s r} \mu_{l}^{r 0}}{\left(\tilde{E}_{r 0}-\hbar \omega^{\prime}\right)\left(\tilde{E}_{s 0}-\frac{1}{2} \tilde{E}_{\alpha^{\prime} 0}-\hbar \omega^{\prime}\right)\left(\tilde{E}_{t \alpha^{\prime}}-\hbar \omega^{\prime}\right)}+\frac{\mu_{j}^{\alpha^{\prime} t} \mu_{k}^{t s} \mu_{i}^{s r} \mu_{l}^{r 0}}{\left(\tilde{E}_{r 0}-\hbar \omega^{\prime}\right)\left(\tilde{E}_{s 0}-\frac{1}{2} \tilde{E}_{\alpha^{\prime} 0}-\hbar \omega^{\prime}\right)\left(\tilde{E}_{t 0}-\frac{1}{2} \tilde{E}_{\alpha^{\prime} 0}\right)}\right) \text {, }
\end{aligned}
$$

since $E_{\alpha^{\prime} 0}=2 \hbar \omega$; this expression is also $i, j$-index symmetric. The twelve distinct terms of Eq. (21) correspond to the twelve different pathways from the initial to the final state in Fig. 6.

\section{Rate expression for laser-modified absorption}

Following derivation of the premultiplier from Eq. (4), $M_{F I}^{(4)}$ is written as

$$
M_{F I}^{(4)}=2^{-\frac{3}{2}} m k k^{\prime}\left(\frac{\hbar c}{\varepsilon_{0} V}\right)^{2} e_{i} e_{j} e_{k}^{\prime} \bar{e}_{l}^{\prime} \chi_{i j k l}^{\alpha^{\prime} 0}\left(\omega^{\prime}\right) .
$$

Through deployment of $M_{F I}^{(2)}$ and $M_{F I}^{(4)}$ with Eq. (6), the rate for laser-modified two-photon absorption is

TABLE IV. As Table II, but for laser-modified two-photon absorption of Fig. 6 .

\begin{tabular}{lcc}
\hline \hline System state $\left|r_{\kappa}^{h}\right\rangle$ & $\left|A_{\kappa} ; n_{\omega}, n_{\omega^{\prime}}\right\rangle$ & Energy $E_{r}$ \\
\hline$|I\rangle \equiv\left|r_{0}^{0}\right\rangle$ & $\left|A_{0} ; 2,1\right\rangle$ & $E_{0}+\hbar\left(2 \omega+\omega^{\prime}\right)$ \\
$\left|r_{1}^{1}\right\rangle$ & $\left|A_{1} ; 1,1\right\rangle$ & $E_{r}+\hbar\left(\omega+\omega^{\prime}\right)$ \\
$\left|r_{1}^{3}\right\rangle$ & $\left|A_{1} ; 2,2\right\rangle$ & $E_{r}+2 \hbar\left(\omega+\omega^{\prime}\right)$ \\
$\left|r_{1}^{9}\right\rangle$ & $\left|A_{1} ; 2,0\right\rangle$ & $E_{r}+2 \hbar \omega$ \\
$\left|r_{2}^{2}\right\rangle$ & $\left|A_{2} ; 0,1\right\rangle$ & $E_{s}+\hbar \omega^{\prime}$ \\
$\left|r_{2}^{4}\right\rangle$ & $\left|A_{2} ; 1,2\right\rangle$ & $E_{s}+\hbar\left(\omega+2 \omega^{\prime}\right)$ \\
$\left|r_{2}^{10}\right\rangle$ & $\left|A_{2} ; 1,0\right\rangle$ & $E_{s}+\hbar \omega$ \\
$\left|r_{2}^{12}\right\rangle$ & $\left|A_{2} ; 2,1\right\rangle$ & $E_{s}+\hbar\left(2 \omega+\omega^{\prime}\right)$ \\
$\left|r_{3}^{5}\right\rangle$ & $\left|A_{3} ; 0,2\right\rangle$ & $E_{t}+2 \hbar \omega^{\prime}$ \\
$\left|r_{3}^{11}\right\rangle$ & $\left|A_{3} ; 0,0\right\rangle$ & $E_{t}$ \\
$\left|r_{3}^{13}\right\rangle$ & $\left|A_{3} ; 1,1\right\rangle$ & $E_{t}+\hbar\left(\omega+\omega^{\prime}\right)$ \\
$|F\rangle \equiv\left|r_{4}^{14}\right\rangle$ & $\left|A_{4} ; 0,1\right\rangle$ & $E_{\alpha^{\prime}}+\hbar \omega^{\prime}$ \\
\hline \hline
\end{tabular}

expressible as

$$
\begin{aligned}
\Gamma_{2 \phi}= & \frac{2^{\frac{1}{2}} \pi \rho I}{c \hbar \varepsilon_{0}} e_{i} e_{j} \bar{e}_{k} \bar{e}_{l}\left[\alpha_{i j}^{(0) \alpha^{\prime} 0} \bar{\alpha}_{k l}^{(0) \alpha^{\prime} 0}+\left(I^{\prime} / c \varepsilon_{0}\right)\right. \\
& \left.\times \alpha_{i j}^{(0) \alpha^{\prime} 0} \bar{\alpha}_{k l}^{(2) \alpha^{\prime} 0}\left(\omega^{\prime}\right)+\left(I^{\prime 2} / 4 c^{2} \varepsilon_{0}^{2}\right) \alpha_{i j}^{(2) \alpha^{\prime} 0}\left(\omega^{\prime}\right) \bar{\alpha}_{k l}^{(2) \alpha^{\prime} 0}\left(\omega^{\prime}\right)\right] .
\end{aligned}
$$

where $\alpha_{i j}^{(0) \alpha^{\prime} 0} \equiv \alpha_{i j}^{\alpha^{\prime} 0}$, in which the superscript 0 denotes a zeroth-order (stimulus beam independent) contribution, and $\alpha_{i j}^{(2) \alpha^{\prime} 0}\left(\omega^{\prime}\right) \equiv e_{k}^{\prime} \bar{e}_{l}^{\prime} \chi_{i j k l}^{\alpha^{\prime} 0}\left(\omega^{\prime}\right)$ corresponds to a contribution quadratic in the electric field of the stimulus beam.

The first term in Eq. (23) corresponds to two-photon absorption, independent of the stimulus beam. The third term signifies absorption in the presence of the elastically forwardscattered stimulus beam. Again it is the second, quantum interference term (linear in $I^{\prime}$ ) that represents the leading correction, and this is the focus in the following subsection.

\section{Three-level system}

As previously, to simplify the expressions, we shall suppose a three-level molecule with states $|0\rangle,|\sigma\rangle$, and $\left|\alpha^{\prime}\right\rangle$, where the latter is the lowest excited state. Identifying the intermediate states $r, s$, and $t$ in Eq. (21) with $0, \alpha^{\prime}$, or $\sigma$ (except where excluded by the restrictions on summation), it is the eleventh term that has the smallest denominator, so that

$$
e_{i} e_{j} \alpha_{i j}^{(2) \alpha^{\prime} 0}\left(\omega^{\prime}\right) \approx-\frac{\mu^{4}}{\Delta \tilde{E}\left(\hbar \omega^{\prime}\right)\left(\tilde{E}_{\sigma 0}-\frac{1}{2} \tilde{E}_{\alpha^{\prime} 0}-\hbar \omega^{\prime}\right)},
$$

on setting $r=0, s=\sigma$, and $t=\sigma$. Here, $E_{\alpha^{\prime} 0}>>\hbar \omega^{\prime}$ and broadly equivalent magnitudes are assumed for the relevant transition dipoles. For two-photon absorption without the input of the stimulus beam, the following result (in which $r=0$ ) will have the greatest contribution:

$$
e_{i} e_{j} \alpha_{i j}^{(0) \alpha^{\prime} 0} \approx-\frac{\mu^{2}}{\hbar \omega^{\prime}}
$$


Typical values for $\Gamma_{2 \phi}$ may be calculated for various offresonant laser intensities; as earlier, setting $\mu=15 \times$ $10^{-30}$ C.m, $\Delta E=-10^{-20} \mathrm{~J}$ and also assuming $\hbar \omega \approx$ $\tilde{E}_{\sigma 0}-\frac{1}{2} \tilde{E}_{\alpha^{\prime} 0}-\hbar \omega^{\prime}=1 \times 10^{-19} \mathrm{~J}$, the rate of two-photon absorption proves to be enhanced by a similar amount to the one-photon case, i.e., $\sim 20 \%$ for an irradiance of $2 \times$ $10^{15} \mathrm{~W} \mathrm{~m}^{-2}$.

\section{SYMMETRY-BASED SELECTION RULES}

The above analysis is sufficiently cast, in general terms, to enable calculations on the strength of the laser-modified effects for any selected one- or two-photon transition in a specific molecule [15]. However many additional principles, and further physical insights, can be gained from a consideration of the selection rules governing these electronic transitions within molecules of a particular symmetry class. Such an analysis is especially valuable because of the variety of different conditions that may arise as a result of the interplay of different optical properties being involved in measurements of the effects.

In general, it can be anticipated that experimental observations of laser-modified absorption will be most readily discernible in molecules and complexes belonging to one of the cubic point groups - those of high symmetry number, with interchangeable Cartesian axes but not necessarily a center of symmetry (a property of the tetrahedral groups, for example). Since this type of high symmetry allows for isotropic polarizability, the molecules will not be oriented by the strong fields of the stimulus radiation, which might otherwise produce complications through photoselection effects. Molecules of such symmetry offer the greatest scope for exploiting states and transitions of varying symmetry. Moreover, cubic symmetry species mostly have electronic excited states similar in structure to the ground state, except for cases where Jahn-Teller effects are prominent.

The procedure for eliciting the interplay of selection rules follows a pattern established in previous work on nonlinear optical processes, see for example Refs. [16-24]. The starting point is a consideration of the irreducible Cartesian tensor components of the polarization and molecular response tensors [13]. An irreducible Cartesian basis affords direct connectivity with the directional properties of the radiation and of the electronic transitions, while also affording a transparent linkage with the symmetry-based irreducible representations of the molecular electronic states, using the rules of angular momentum coupling as the following will demonstrate.

\section{A. Symmetry of laser-modified single-photon absorption}

Returning to the rate expression for laser-modified singlephoton absorption, namely Eq. (15), we now identify the connectivity of the relevant irreducible components of the radiation and material tensors. Of present interest are $\mu_{i}^{(0) \alpha 0}$, representing Cartesian components of the electric dipole transition moment due to resonant absorption-formally a rank-1 polar tensor-and $\mu_{i}^{(2) \alpha 0}\left(\omega^{\prime}\right)$, components of another rank-1 polar tensor representing the influence of the stimulus radiation. The latter, from its defining equation given earlier, is now recast as

$$
\mu_{i}^{(2) \alpha 0}\left(\omega^{\prime}\right) \equiv\left[\boldsymbol{\beta}^{\alpha 0}\left(\omega^{\prime}\right): \mathbf{S}\right]_{i},
$$

where $\mathbf{S} \equiv e_{j}^{\prime} \bar{e}_{k}^{\prime}$ is an even parity rank-2 tensor signifying the second-order electric field influence of the stimulus beam, and $\beta_{i j k}^{\alpha 0}\left(\omega^{\prime}\right)$ are components of $\boldsymbol{\beta}^{\alpha 0}\left(\omega^{\prime}\right)$, the rank-3 tensor whose symmetry properties follow from Eq. (11), and whose explicit components are defined by Eq. (13). In general, the latter lacks any index symmetry and, as a consequence, the tensor may be decomposed into irreducible parts of weight $0^{-}, 1^{-}, 2^{-}$, and $3^{-}$, all of odd parity, i.e.,

$$
\boldsymbol{\beta}^{\alpha 0}=\left[\boldsymbol{\beta}^{\alpha 0}\right]^{(0-)}+\left[\boldsymbol{\beta}^{\alpha 0}\right]^{(1-)}+\left[\boldsymbol{\beta}^{\alpha 0}\right]^{(2-)}+\left[\boldsymbol{\beta}^{\alpha 0}\right]^{(3-)},
$$

now suppressing the frequency dependence, for clarity. Also, the contributions of specific weights and parity are designated by bracketed superscripts outside the square brackets, to clarify their distinction from the interaction orders introduced earlier. In sequence, from left to right, the four terms of Eq. (27) (all embedded in rank-3 tensor space) are defined as follows: a pseudoscalar; a polar vector; an odd-parity, symmetric, and traceless second-rank tensor; and a polar traceless and fully index-symmetric third rank tensor. The detailed form of components for each of these contributions has long been established and given elsewhere [13]: for present symmetry analysis purposes, their explicit formulae are not required.

A similar reduction of the stimulus tensor into components yields

$$
\mathbf{S}=[\mathbf{S}]^{(0+)}+[\mathbf{S}]^{(1+)}+[\mathbf{S}]^{(2+)},
$$

where successive terms on the right transform as: a scalar; an axial vector; an even-parity traceless and symmetric secondrank tensor. In this instance, it is worth presenting the explicit form of the corresponding components under two separate conditions: for a linearly polarized stimulus beam

$$
[\boldsymbol{S}]_{i j}^{(0+)}=\frac{1}{3} \delta_{i j}, \quad[\boldsymbol{S}]_{i j}^{(1+)}=0, \quad[\boldsymbol{S}]_{i j}^{(2+)}=e_{i}^{\prime} e_{j}^{\prime}-\frac{1}{3} \delta_{i j},
$$

and a circularly polarized off-resonant laser

$[\boldsymbol{S}]_{i j}^{(0+)}=\frac{1}{3} \delta_{i j}, \quad[\boldsymbol{S}]_{i j}^{(1+)}=\frac{i}{2} \varepsilon_{i j k} \hat{k}_{k}^{\prime}, \quad[\boldsymbol{S}]_{i j}^{(2+)}=\frac{1}{6} \delta_{i j}-\frac{1}{2} \hat{k}_{i}^{\prime} \hat{k}_{j}^{\prime}$.

On comparison of the two cases, a particularly significant difference is the absence of a weight $-1^{+}$component for linear (plane) polarization.

Now returning to Eq. (26), on using the rules of parity and angular momentum coupling, defined by $\left|j_{1}-j_{2}\right| \leqslant j \leqslant$ $j_{1}+j_{2}$, and recognizing that the result must be a vector, which carries a weight- 1 contribution only, the following is determined:

$$
\begin{aligned}
{\left[\boldsymbol{\beta}^{\alpha 0}: \mathbf{S}\right]=} & \frac{\left[\boldsymbol{\beta}^{\alpha 0}\right]^{(0-)}[\mathbf{S}]^{(1+)}+\left[\boldsymbol{\beta}^{\alpha 0}\right]^{(1-)}[\mathbf{S}]^{(0+)}}{}+\underline{\left[\boldsymbol{\beta}^{\alpha 0}\right]^{(1-)}[\mathbf{S}]^{(1+)}}+\left[\boldsymbol{\beta}^{\alpha 0}\right]^{(1-)}[\mathbf{S}]^{(2+)} \\
& +\underline{\left[\boldsymbol{\beta}^{\alpha 0}\right]^{(2-)}[\mathbf{S}]^{(1+)}}+\left[\boldsymbol{\beta}^{\alpha 0}\right]^{(2-)}[\mathbf{S}]^{(2+)} \\
& +\left[\boldsymbol{\beta}^{\alpha 0}\right]^{(3-)}[\mathbf{S}]^{(2+)}
\end{aligned}
$$

where the underlined terms denote contributions that disappear if the stimulus beam is plane polarized. For such a case, the weight $-0^{-}$contributions are absent from the material response tensor. 
Transitions that are conventionally one-photon allowed will always display a degree of rate alteration by the laser-modified mechanism, since the corresponding rate correction terms will also be symmetry allowed. However, certain other singlephoton absorption processes, conventionally forbidden (the corresponding upper levels often being termed dark states) may furthermore become allowed, if they have the necessary symmetry character. Of these photoactivated transitions, some would also be allowed by the selection rules for two-photon absorption (from a beam of half the optical frequency), but others would not be allowed by either single-photon or two-photon processes by convention. This can be understood through the following detail.

It is clear that one-photon allowed processes require threephoton events to be symmetry permissible too, although the converse is not always true. As a result, unattainable dark states become active under certain symmetries. For example, $A_{2}$ electronic transitions in $C_{2 v}$ point groups (e.g., water molecules), or $\sum_{u}^{-}$in $D_{\infty h}$ groups (e.g., carbon dioxide), contain weight- $0^{-}$and weight- $2^{-}$contributions but no weight$1^{-}$. Physically, this means that one-photon absorption from the totally symmetric ground state to electronic excited states of these symmetries, usually forbidden, become perfectly conceivable through the laser-modified mechanism. Moreover, although $A_{2}$ transitions in $C_{2 v}$ molecules are formally (though very weakly) electric quadrupole allowed, even in the absence of the stimulus beam, the latter case of $\sum_{u}^{-}$transitions in $D_{\infty h}$ groups typifies a class of transitions that is formally both electric dipole and quadrupole forbidden, becoming allowed through the involvement of the stimulus beam. In both of these examples the throughput stimulus beam engages with the absorption of the resonant pump radiation irrespective of the former beam's polarization. Other instances, where transitions can only be activated by circularly polarized stimulus lightthose containing weight- $0^{-}$contributions singly-include $A_{2}$ transitions in $T_{d}$ (e.g., methane).

\section{B. Symmetry of laser-modified two-photon absorption}

A similar analysis can be undertaken for the case of twophoton absorption, using Eq. (23) as the starting point. Here, the rank-2 even-parity tensors of $\alpha_{i j}^{(0) \alpha^{\prime} 0}$ and $\alpha_{i j}^{(2) \alpha^{\prime} 0}\left(\omega^{\prime}\right)$ are the central factors. The latter may be recast as

$$
\alpha_{i j}^{(2) \alpha^{\prime} 0}\left(\omega^{\prime}\right) \equiv\left[\chi^{\alpha^{\prime} 0}\left(\omega^{\prime}\right): \mathbf{S}\right]_{i j},
$$

where $\chi^{\alpha^{\prime} 0}\left(\omega^{\prime}\right)$, the rank-4 tensor of Eq. (21), may be decomposed into irreducible parts of weight $0^{+}, 1^{+}, 2^{+}, 3^{+}$, and $4^{+}$, so that

$$
\begin{aligned}
\chi^{\alpha^{\prime} 0}= & {\left[\chi^{\alpha^{\prime} 0}\right]^{(0+)}+\left[\chi^{\alpha^{\prime} 0}\right]^{(1+)}+\left[\chi^{\alpha^{\prime} 0}\right]^{(2+)} } \\
& +\left[\chi^{\alpha^{\prime} 0}\right]^{(3+)}+\left[\chi^{\alpha^{\prime} 0}\right]^{(4+)}
\end{aligned}
$$

where the final term denotes a natural fourth rank tensor (i.e., a tensor whose weight equals its rank). Again using the rules of parity, and recognizing that the result is a rank-2 tensor containing weight- 0 and weight- 2 contributions, the following is found:

$$
\begin{aligned}
{\left[\chi^{\alpha^{\prime} 0}: \mathbf{S}\right]=} & {\left[\chi^{\alpha^{\prime} 0}\right]^{(0+)}[\mathbf{S}]^{(0+)}+\left[\chi^{\alpha^{\prime} 0}\right]^{(0+)}[\mathbf{S}]^{(2+)} } \\
& +\underline{\left[\chi^{\alpha^{\prime} 0}\right]^{(1+)}[\mathbf{S}]^{(1+)}+\left[\chi^{\alpha^{\prime} 0}\right]^{(1+)}[\mathbf{S}]^{(2+)}}
\end{aligned}
$$

$$
\begin{aligned}
& +\left[\chi^{\alpha^{\prime} 0}\right]^{(2+)}[\mathbf{S}]^{(0+)}+\underline{\left[\chi^{\alpha^{\prime} 0}\right]^{(2+)}[\mathbf{S}]^{(1+)}} \\
& +\left[\chi^{\alpha^{\prime} 0}\right]^{(2+)}[\mathbf{S}]^{(2+)}+\underline{\left[\chi^{\alpha^{\prime} 0}\right]^{(3+)}[\mathbf{S}]^{(1+)}} \\
& +\left[\chi^{\alpha^{\prime} 0}\right]^{(3+)}[\mathbf{S}]^{(2+)}+\left[\chi^{\alpha^{\prime} 0}\right]^{(4+)}[\mathbf{S}]^{(2+)}
\end{aligned}
$$

where the underlined terms denote contributions that disappear if the stimulus beam is plane polarized. Under the latter conditions, all weight contributions from $\chi^{\alpha^{\prime} 0}$ still feature in the result so that, in contrast to the single-photon case, there is no experimental advantage or discriminatory power to be gained by using a circularly polarized laser.

Analogous to the one-photon case, all transitions that are conventionally two-photon allowed will display some rate alteration by the laser-modified mechanism. Moreover, when two-photon absorption is forbidden, for certain symmetries it is possible for the optical activation of dark states relating to two-photon absorption. To determine electronic transitions that are usually forbidden, but which become allowed through laser-modified two-photon methods, we focus on weights $1^{+}, 3^{+}$, and $4^{+}$contributions as determined from Eq. (34) (weights $0^{+}$and $2^{+}$being omitted since they already support two-photon absorption). Examples here include $A_{2}$ transitions in $C_{3 v}$ point groups (e.g., ammonia), $\sum^{-}$in $C_{\infty v}$ groups (e.g., carbon monoxide), and $A_{2}^{\prime \prime}$ in $D_{3 h}$ (e.g., boron trifluoride). A most intriguing feature arises when one-photon absorption is also symmetry allowed. Physically, this denotes a state previously unavailable by two-photon absorption, but allowed by a single photon equivalent, which becomes accessible via laser-modified two-photon absorption. The boron trifluoride example given above is an example of one such case.

\section{DISCUSSION}

Although other methods have recently been proposed to enhance light absorption, including those based on plasmonics [25-29] and geometry [30-33], it is interesting to reflect that the mechanisms we have identified, for essentially passive effects on absorption being produced by a nonresonant laser beam, seem to have escaped earlier notice. It may be surmised that the reason is the more common semiclassical formulation of theory for single- and two-photon absorption processes. When such processes are described on the basis of linear and quadratic response to an optical field cast as $E_{0} \cos \omega t$, the higher-order cubic and quartic terms are generally dispensed with on the supposition that they signify only negligible corrections, which is true, if the beam from which the absorption occurs is the only light present. The mechanisms that we have described become important precisely because they can be brought into effect by a secondary beam, designedly off-resonant but also at a higher intensity than the resonant radiation.

As has been shown, the presence of off-resonant light can significantly modify absorption rates, and we have identified specific criteria for the relative positioning of energy levels that will support either rate enhancement or diminution. In both such cases it can be anticipated that lines in the ultraviolet or visible spectrum, associated with electronic transitions of appropriate symmetry, will exhibit a modification of intensity, also associated with a change in the corresponding excited state lifetime. Such an effect might be simplest to measure through 
a change in absorptivity at the red edge of the absorption band, where there will be fewer complications due to the excitation of vibrational modes in the upper electronic state. The magnitude of the change in absorption rate, responsible for each of these effects, will be linearly dependent on the stimulus beam intensity, affording a ready means to experimentally verify operation of the stimulus mechanism.

Notably, it has also emerged that such stimulus beam effects can also promote excitation to dark, i.e., normally forbidden states. In this respect this form of optical nonlinearity may represent a significant opportunity for exerting optical control in the quantum regime. Consider, for example, a nanocavity in which one photon of the stimulus beam is present at a given time. A molecule of suitable symmetry (or an atom) within the cavity should then in principle exhibit a different degree of coupling with other, resonant radiation, compared to the behavior when no stimulus photons are present. This potentially affords a means to switch the resonant absorption on or off. Since this effect operates, despite a stimulus photon remaining present in the cavity, it represents a kind of quantum nondemolition measurement [34] on that photon. The rich interplay of nonlinear optics, quantum optics, polarization, and symmetry principles underlying all of these potentialities suggest there is plenty more scope to exploit and develop the theory we have delivered.

\section{ACKNOWLEDGMENT}

The authors are grateful to the Leverhulme Trust for funding this research.
[1] W. I. Fushchich and A. G. Nikitin, J. Phys. A 25, L231 (1992).

[2] D. S. Bradshaw and D. L. Andrews, J. Phys. Chem. A 113, 6537 (2009).

[3] D. S. Bradshaw and D. L. Andrews, Phys. Rev. A 81, 013424 (2010).

[4] J. M. Leeder, D. S. Bradshaw, and D. L. Andrews, J. Phys. Chem. B 115, 5227 (2011).

[5] R. G. Woolley, Adv. Quant. Chem. 32, 167 (1998).

[6] C. Gerry and P. Knight, Introductory Quantum Optics (Cambridge University Press, Cambridge, 2005).

[7] D. P. Craig and T. Thirunamachandran, Molecular Quantum Electrodynamics: An Introduction to Radiation-Molecule Interactions (Dover, Mineola, 1998).

[8] C. H. Wilcox, Perturbation Theory and its Applications in Quantum Mechanics (Wiley Chapman and Hall, New York, 1966).

[9] L. Mandel and E. Wolf, Optical Coherence and Quantum Optics (Cambridge University Press, Cambridge, 1995).

[10] R. D. Jenkins, D. L. Andrews, and L. C. Dávila Romero, J. Phys. B: At. Mol. Opt. Phys. 35, 445 (2002).

[11] D. L. Andrews and D. S. Bradshaw, Eur. J. Phys. 30, 239 (2009).

[12] C. D. Godsil and G. F. Royle, Algebraic Graph Theory (SpringerVerlag, New York, 2001).

[13] D. L. Andrews and P. Allcock, Optical Harmonics in Molecular Systems (Wiley-VCH, Weinheim, 2002).

[14] W. Demtröder, Laser Spectroscopy: Basic Concepts and Instrumentation (Springer-Verlag, Berlin, 2003).

[15] W. M. McClain, Symmetry Theory in Molecular Physics with Mathematica (Springer-Verlag, New York, 2008).

[16] D. L. Andrews and N. P. Blake, J. Phys. A: Math. Gen. 22, 49 (1989).
[17] J. Zyss and S. Brasselet, J. Nonlinear Opt. Phys. Mater. 7, 397 (1998).

[18] S. Brasselet and J. Zyss, J. Opt. B: Quantum Semiclass. Opt. 15, 257 (1998).

[19] C. Andraud, T. Zabulon, A. Collet, and J. Zyss, Chem. Phys. 245, 243 (1999).

[20] R. Piron, S. Brasselet, D. Josse, J. Zyss, G. Viscardi, and C. Barolo, J. Opt. B: Quantum Semiclass. Opt. 22, 1276 (2005).

[21] J. Zyss, Proc. SPIE 7029, 704002 (2008).

[22] D. L. Andrews, Phys. Rev. A 81, 033825 (2010).

[23] S. N. A. Smith and D. L. Andrews, J. Phys. A: Math. Gen. 44, 395001 (2011).

[24] T. Bancewicz, J. Math. Chem. 50, 1570 (2012).

[25] Y. A. Akimov, W. S. Koh, and K. Ostrikov, Opt. Express 17, 10195 (2009).

[26] W. Wang, S. Wu, K. Reinhardt, Y. Lu, and S. Chen, Nano Lett. 10, 2012 (2010).

[27] P. Zilio, D. Sammito, G. Zacco, M. Mazzeo, G. Gigli, and F. Romanato, Opt. Express 20, A476 (2012).

[28] J. H. Vella and A. M. Urbas, J. Phys. Chem. C 116, 17169 (2012).

[29] P. Spinelli and A. Polman, Opt. Express 20, A641 (2012).

[30] H. You, S. M. Hendrickson, and J. D. Franson, Phys. Rev. A 78, 053803 (2008).

[31] X. Li, J. van Embden, J. W. M. Chon, and M. Gu, Appl. Phys. Lett. 94, 103117 (2009).

[32] K. Saha, V. Venkataraman, P. Londero, and A. L. Gaeta, Phys. Rev. A 83, 033833 (2011).

[33] L.-C. Zhang, G. Yang, K. Wang, M. Fu, Y. Wang, H. Long, and P.-X. Lu, Opt. Commun. 291, 395 (2013).

[34] P. Grangier, J. A. Levenson, and J. P. Poizat, Nature (London) 396, 537 (1998). 